\title{
Patterned Activity via Spinal Dorsal Quadrant Inputs Is Necessary for the Formation of Organized Somatosensory Maps
}

\author{
Neeraj Jain, ${ }^{1}$ Pamela S. Diener, ${ }^{2,3}$ Jacques-Olivier Coq, ${ }^{1}$ and Jon H. Kaas ${ }^{1}$ \\ ${ }^{1}$ Department of Psychology, Vanderbilt University, Nashville, Tennessee 37240, ${ }^{2}$ Department of Pharmacology and Experimental Therapeutics, University \\ of Maryland, School of Medicine, Baltimore, Maryland 21201, and '3Department of Physical Therapy, Marymount University, School of Health Professions, \\ Arlington, Virginia 22207
}

\begin{abstract}
The normal development of the somatosensory system requires intact sensory inputs from the periphery during a critical window of time early in development. Here we determined how the removal of only part of the ascending spinal inputs early in development affects the anatomical and neurophysiological development of the somatosensory system. We performed spinal overhemisections in rat pups at $\mathrm{C} 3 / \mathrm{C} 4$ levels on the third day after birth. This procedure hemisects the spinal cord on one side and transects the dorsal funiculus on the other side. When the rats were 6-8 months old, the responsiveness and somatotopy of the primary somatosensory cortex (S1) contralateral to the hemisection were determined using standard multiunit mapping techniques. Sections of the flattened cortex were processed for cytochrome oxidase activity, Nissl substance, or myelin. We found that histologically apparent modules that are normally present in the regions of the forepaw and the hindpaw representations were absent, whereas the lateral barrel field representing the face was completely normal. The neurons in the forepaw regions of $\mathrm{S} 1$ either did not respond to the stimulation of the skin of any region of the body or responded to the stimulation of the upper arm afferents that enter the spinal cord rostral to the site of the lesion. The results show that a lack of normal sensory inputs via ascending pathways in the dorsal spinal cord during early development results in massive anatomical and neurophysiological abnormalities in the cortex. Intact crossed spinothalamic pathways are unable to support the normal development of the forepaw barrels.
\end{abstract}

Key words: somatosensory cortex; development; cuneate nucleus; rat; spinal cord injury; plasticity

\section{Introduction}

Injuries that result in disruption of normal sensory inputs during the critical period of development lead to severe and irreversible changes in the topographic maps, connection patterns, and functioning of the mature brain. Somatotopic maps in the primary somatosensory cortex of rats develop abnormally after complete loss of sensory inputs by neonatal or prenatal amputation of a limb, removal of the facial whiskers, or nerve transections (Woolsey and Wann, 1976; Jensen and Killackey, 1987; Killackey and Dawson, 1989; Waters et al., 1990; Rhoades et al., 1997). However, somatosensory inputs segregate and ascend in multiple tracts in the spinal cord, including uncrossed fibers in the dorsal columns and dorsolateral spinal cord and the crossed spinothalamic pathways in the ventral and ventrolateral spinal cord. The relative contribution of sensory inputs via these different spinal pathways in the development of the normal somatosensory system is not clear. To determine how different input pathways

Received Feb. 27, 2003; revised Sept. 4, 2003; accepted Sept. 10, 2003.

This work was supported by National Institutes of Health Grant NS 16446 (J.H.K.) and Christopher Reeve Paralysis Foundation Grants JB1-9803 (N.J.) and DA1-9802 (P.S.D.). We thank Drs. Christine Collins and V. Rema for helpful comments on this manuscript.

Correspondence should be addressed to Neeraj Jain at his present address: National Brain Research Centre, Nainwal Mode, Manesar (Haryana), 122 050, India. E-mail: neeraj.jain@nbrc.ac.in.

Copyright $\odot 2003$ Society for Neuroscience $\quad$ 0270-6474/03/2310321-10\$15.00/0 contribute to the formation of the somatotopic patterns in the brain, we determined the effects of spinal overhemisections at C3/C4 $3 \mathrm{~d}$ after birth on the physiological and anatomical organization of the rat primary somatosensory cortex (S1). This procedure, generally used to study the effects of spinal cord injury on the development of motor behavior and regenerative growth within the spinal cord, cuts all the ascending and descending fiber pathways on the hemisection side and the ascending uncrossed dorsal column somatosensory afferents and the descending dorsal corticospinal motor axons on the oversection side. The procedure, thus, deprives somatosensory cortex ipsilateral to the hemisection of somatosensory inputs from most of the contralateral body via both dorsal columns and spinothalamic pathways. Only inputs from the face and a few from the upper arm entering the spinal cord rostral to the lesion remain intact. In contrast, the cortex ipsilateral to the oversection maintains access to the crossed spinothalamic pathway but loses inputs via uncrossed pathways in the dorsal spinal cord.

We used this preparation to address the following questions about the development of the somatosensory pathway in rats. (1) Will cortex deprived of activating inputs from the dorsal spinal cord soon after birth develop responsiveness to the intact spinothalamic inputs? This is not the case in adult rats in which S1 cortex appears to be completely dependent on the dorsal column 
pathway for activation (Jain et al., 1995) but might be occurring in developing monkeys after dorsal column lesions (Jain et al., 2001b). (2) Would the barrel-like modular pattern of thalamocortical terminals that reflects specific body parts (e.g., digits and pads of forepaw) develop in portions of S1 deprived of dorsal column but not spinothalamic sources of activation? Such modules do not develop when both sources of inputs are removed by limb amputation early in development (Dawson and Killackey, 1987; Killackey and Dawson, 1989; Waters et al., 1990; Pearson et al., 1999). (3) Will surviving afferents from the face and upper arm activate larger than normal territories in S1, including portions of S1 deprived of normal somatosensory inputs? Such an expansion of activated territory does not occur to any notable extent after dorsal column lesions in adult rats (Jain et al., 1995) (but see Wall and Egger, 1971), but it does in adult monkeys after dorsal column lesions or limb loss (Florence and Kaas, 1995; Jain et al., 1997). To address these questions, we used multiunit mapping techniques to study the responsiveness of neurons and histological methods to study the modular organization of S1.

Parts of this work have been published previously in abstract form (Jain et al., 2000b).

\section{Materials and Methods}

All animal procedures followed National Institutes of Health guidelines and were approved by Vanderbilt University and University of Maryland School of Medicine Animal Care and Use Committees.

Cervical spinal cord injury. Timed-pregnant multiparous Sprague Dawley rats (Zivic Miller Laboratories, Zelienople, PA) were observed several times per day for the birth of pups. The day of birth was assigned as postnatal day $1(\mathrm{P} 1)$. Newborn pups $(n=23)$ were anesthetized by hypothermia on P3. Viewing through a surgical microscope, each rat's skin along the back of the neck was incised with a number 11 surgical blade, and muscles were separated and retracted in layers to reveal the spinal column. A laminectomy was performed at the C3 spinal level. The dural sheath overlying the C3 segment of spinal cord was slit with a number 11 surgical blade, and the dorsal columns and dorsal rootlets were visualized. Using iridectomy scissors, an overhemisection lesion was made. This lesion damages the dorsal funiculus, bilaterally, as well as the lateral and ventral funiculi and gray matter on the right side of the $\mathrm{C} 3$ spinal segment. Next, Sil-tec, a synthetic material to substitute for the absence of dural membrane (Technical Products, Decatur, GA) was inserted over the lesion cavity and topped by a saline-soaked gel foam pledget. The muscles and overlying skin were then sutured in layers with 6.0 silk. The rats recovered in a warm environment and were given a subcutaneous prophylactic dose of Bicillin (Wyeth Laboratories, Philadelphia, PA) before being returned to their respective mothers. The spinal-injured rat pups were then randomly selected for one of the two experimental groups. One group of postsurgical rat pups was exposed daily to a variety of enriched sensorimotor environments, whereas the other group of injured rats developed within the confines of standard laboratory housing. The development of forelimb motor activity was assessed in all rats during their first 2-2.5 months of life (Diener, 2002). When the rats were $6-8$ months old, we randomly selected a total of six rats, of either sex, from the two groups for present studies. Four of the rats (99-27, 99-29, 99-33, and 99-41) were from the enriched group, and two (99-35 and 99-43) from the non-enriched group. However, no differences were found between the two groups in this study, and the results from both groups are presented together. We also used six hemispheres from three normal rats for comparison.

Multiunit mapping. Rats were anesthetized with urethane $(250 \mathrm{mg} / \mathrm{kg}$, i.p.) supplemented with ketamine $(90 \mathrm{mg} / \mathrm{kg})$ as needed. A craniotomy was made to expose the S1 region contralateral to the hemisection (left hemisphere), the dura was opened, and the brain was covered with silicone to prevent desiccation. Cortex was mapped using standard multiunit mapping techniques using parylene-coated tungsten microelectrodes (1 M $\Omega$ at $1 \mathrm{kHz}$; MicroProbe, Potomac, MD). The electrode penetration sites were marked on a magnified photograph of the cortical surface using surface vasculature as a guide. Receptors in the skin were stimulated using hand-held stimulators such as brushes and wooden probes. Responses to the stimulation of deep receptors and movements of the muscles and joints were also determined. The person mapping the receptive fields was blind to the location of the electrode in the brain. The receptive fields were drawn on the drawings of body outlines. Toward the end of the mapping session, microlesions were made at selected sites by passing cathodal current ( $10 \mu \mathrm{A}$ for $10 \mathrm{sec}$ ) to aid in aligning the electrophysiological map with the anatomical map of the barrel field (for details, see Jain et al., 1995).

Cholera toxin subunit B linked to horseradish peroxidase injections. To assess the extent and source of the remaining inputs, if any, attributable to an incomplete lesion, we injected transganglionic neuronal tracer cholera toxin subunit B linked to horseradish peroxidase (B-HRP) (List Biologic, Campbell, CA) at multiple sites (Jain et al., 1995, 1997) in the skin of the arms of three rats (99-35, 99-41, and 99-43). The injections were made at $8-13$ sites all over the arm $(0.1 \% \mathrm{~B}-\mathrm{HRP}, \sim 4 \mu \mathrm{l} / \mathrm{site})$. Series of sections from the spinal cord and lower medulla were processed for TMB reaction to visualize HRP (Gibson et al., 1984). Presence of the TMB reaction product in the cuneate nucleus was used to determine the extent and source of the intact dorsal column inputs, if any (Jain et al., 1995, 1997).

Perfusion and histological processing of the brain. At the end of the mapping sessions, the rats were perfused transcardially with buffered saline, followed by buffered $2 \%$ paraformaldehyde and then by buffered $2 \%$ paraformaldehyde containing $10 \%$ sucrose. The brain and spinal cord were removed. Cortex was separated from the brainstem and flattened between glass slides. The tissue was cryoprotected in $30 \%$ sucrose and sectioned frozen on a sliding microtome. The cortex was sectioned at 60 or $70 \mu \mathrm{m}$ parallel to the pial surface. Spinal cord was sectioned at 40 $\mu \mathrm{m}$ in the horizontal plane, and lower brainstem was sectioned at 32 or $34 \mu \mathrm{m}$ in a coronal plane.

Series of sections from the cortex were stained for Nissl substance, cytochrome oxidase (CO) activity (Wong-Riley, 1979), or myelin (Jain et al., 1998, 2001a). Series of alternate sections from the medulla were stained for CO activity and for TMB reaction (Gibson et al., 1984). All of the sections from the spinal cord were stained for TMB reaction.

Reconstruction of the lesion site. Drawings of the sections of the spinal cord were made using a dark-field microscope equipped with camera lucida. The region of the spinal cord with the lesion was reconstructed in a coronal plane using the midline as the reference point.

\section{Results}

Recordings were obtained from S1 in the left hemisphere that was deprived of the dorsal columns but not the spinothalamic inputs. The results show that this loss produced by spinal overhemisections early in development results in complete lack of responses to the deafferented parts of the skin, a limited expansion of the zone of cortex responsive to intact arm inputs, and a failure of development of the normal modular histological appearance of the $\mathrm{S} 1$.

\section{The normal modular pattern in S1 cortex and the use of myelin stain to reveal the pattern}

We stained sections of the flattened cortex for three different histological markers (CO activity, Nissl substance, and myelin) to reveal the effects of spinal hemisections on the anatomical development of the S1 cortex (Fig. 1). Sections from the cortex of three normal rats were used for comparison. The organization of the normal barrel pattern in rodents, first discovered in Nissl-stained material (Woolsey and Van der Loos, 1970; Woolsey et al., 1975) (Fig. $1 \mathrm{~B}$ ), reveals each of the barrels as a cell-dense ring enclosing a relatively cell-sparse center that has been dubbed the barrel hollow. However, metabolic markers CO (Wong-Riley and Welt, 1980) (Fig. 1C) and succinic dehydrogenase (Dawson and Killackey, 1987) are the more commonly used markers because of 

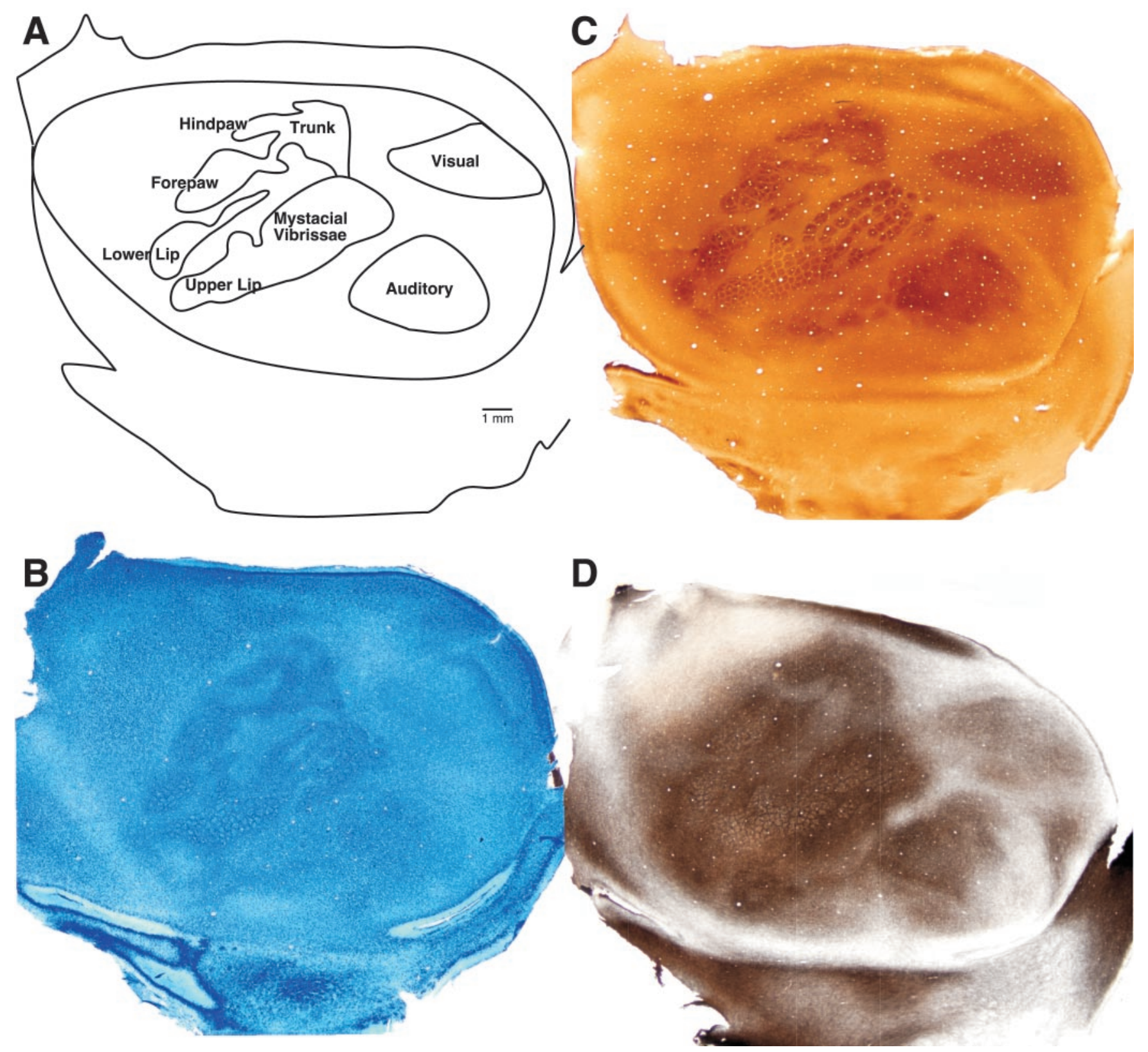

Figure 1. The organization of $\mathrm{S} 1$ of rats as revealed in Niss--, $\mathrm{CO}$-, and myelin-stained sections of the flattened cortex. $A$, An outline diagram showing the locations of the mystacial vibrissae, lips, forepaw, hindpaw, and trunk representations in S1. Primary visual and auditory cortices are marked for reference. $B$, Photomontage of Nissl-stained sections from the flattened cortex of a rat showing barrels and modules in the S1. The barrels appear with a cell-sparse "hollow" surrounded by cell-dense "walls." C, In CO-stained sections, the barrels are visible as CO-dark modules surrounded by $\mathrm{C} O$-light septa. The distinct modular pattern is visible in the vibrissae, lips, forepaw, and hindpaw regions. In the trunk region, the staining is uniformly dark. The $\mathrm{CO}$-dark patches lateral to $\mathrm{S1}$ correspond to second somatosensory (S2) and parietal ventral (PV) areas. D, We also stained sections of the flattened cortex for myelin. In myelin-stained sections through the middle layers of the cortex, the barrels appear as myelin-light patches, whereas the septa are darkly stained. The regions of the trunk representation are uniformly stained as for $C 0$ and Nissl. In the deeper layers, the staining pattern is reversed with myelin-dark barrels and myelin-light septa (see Fig. 6).

the high contrast that they provide. The normal organization of the rat S1 has been described previously (Dawson and Killackey, 1987). Briefly, cytochrome oxidase stains the area S1 darkly compared with the surrounding cortex (Fig. 1). Within S1, discrete $\mathrm{CO}$-dark cell clusters (modules or barrels) are seen that are surrounded by $\mathrm{CO}$-light regions called septa. The barrels are organized in groups that correspond to the representations of different body parts. The lateralmost group, known as posteromedial barrel subfield (PMBSF), represents whiskers on the face. Each of the large caudal barrels in PMBSF corresponds to the representations of a single mystacial whisker (Woolsey et al., 1975). Direct thalamocortical inputs from each whisker are highly restricted to a specific barrel in S1. The more rostral barrels in this subfield represent sinus hairs on the upper lip and the buccal pad (Welker, 1976). Medial to PMBSF in the rostral S1, a small arch of barrels corresponds to the representation of the hairs of the lower lip. A more medial group of modules represents the digits and the pads of the forepaw (Dawson and Killackey, 1987; Waters et al., 1995), and another medialmost group corresponds to the representation of the parts of the hindpaw (Dawson and Killackey, 1987). Caudal to the forepaw and the hindpaw representations, a zone of more uniform staining represents the trunk. The trunk representation is connected to the forepaw and hindpaw subfields by narrow rostrocaudal CO-dark strips corresponding to the repre- 
sentations of the arm and leg. Thus, a complete pattern of histological isomorphs of body parts can be clearly visualized in the S1 cortex (Fig. 1).

In addition to these often used $\mathrm{CO}$ and Nissl stains, we also stained sections of the flattened cortex for myelin to reveal the barrels (Fig. 1D). The barrels and clusters were clearly seen in myelin-stained sections from the flattened cortex. In the middle layers of the cortex, these modules stained lightly for myelin, whereas the interbarrel regions or septa were darkly stained. In the infragranular layers, however, the pattern of staining for myelin was reversed. The modules stained darker, and the septa were light in appearance (see Fig. 6). The barrels were clearly visible in all regions of PMBSF, lower jaw, forepaw, and hindpaw, whereas the parts of the S1 cortex representing the trunk, arm, and leg were more evenly stained, as seen in the CO- or Nissl-stained preparations.

\section{The extent of the spinal lesions}

Spinal cord lesions were made to disrupt the right half of the spinal cord and the left dorsal funiculus (Figs. 2-5). Drawings of the horizontal sections of the spinal cord were used to reconstruct the lesion site in a coronal view. These reconstructions showed that, in all of the rats, the right dorsal quadrant was completely destroyed (Figs. $2 C-5 C$ ), except for small sparing of the ventralmost dorsal funiculus in rats 99-27 and 99-35 (Fig. 4C). In four of the rats (Fig. $2 C, D$, rat 99-29; Fig. $3 C, D$, rat 99-33; Fig. 5C, rat 99-43) (rat 99-41, data not shown), there was complete interruption of the dorsal funiculus on both sides and the right lateral funiculus. In these rats, the right ventral quadrant was also nearly completely interrupted except for small variable sparing near the midline. In the remaining two rats, 99-27 and 99-35 (Fig. 4C), the right lateral funiculus was completely destroyed. In rat 99-27, there was a small sparing of the ventralmost dorsal funiculus and the medial half of the right ventral quadrant. In rat 99-35, a small ventral portion of the right dorsal funiculus and nearly all of the left dorsal funiculus was spared, whereas the ventral quadrant had some sparing in the dorsal portion.

\section{Changes in the barrel pattern in the S1 cortex}

Histological sections of the cortex contralateral to the spinal cord hemisection were examined in each of the rats. This cortex was deprived of inputs via dorsal quadrant afferents, whereas the spinothalamic afferents remained intact. In four of the rats (Fig. 2, rat 99-29; Fig. 3, rat 99-33) (rats 99-27 and 99-41, data not shown) sections of the flattened cortex were stained for $\mathrm{CO}$ activity, whereas sections
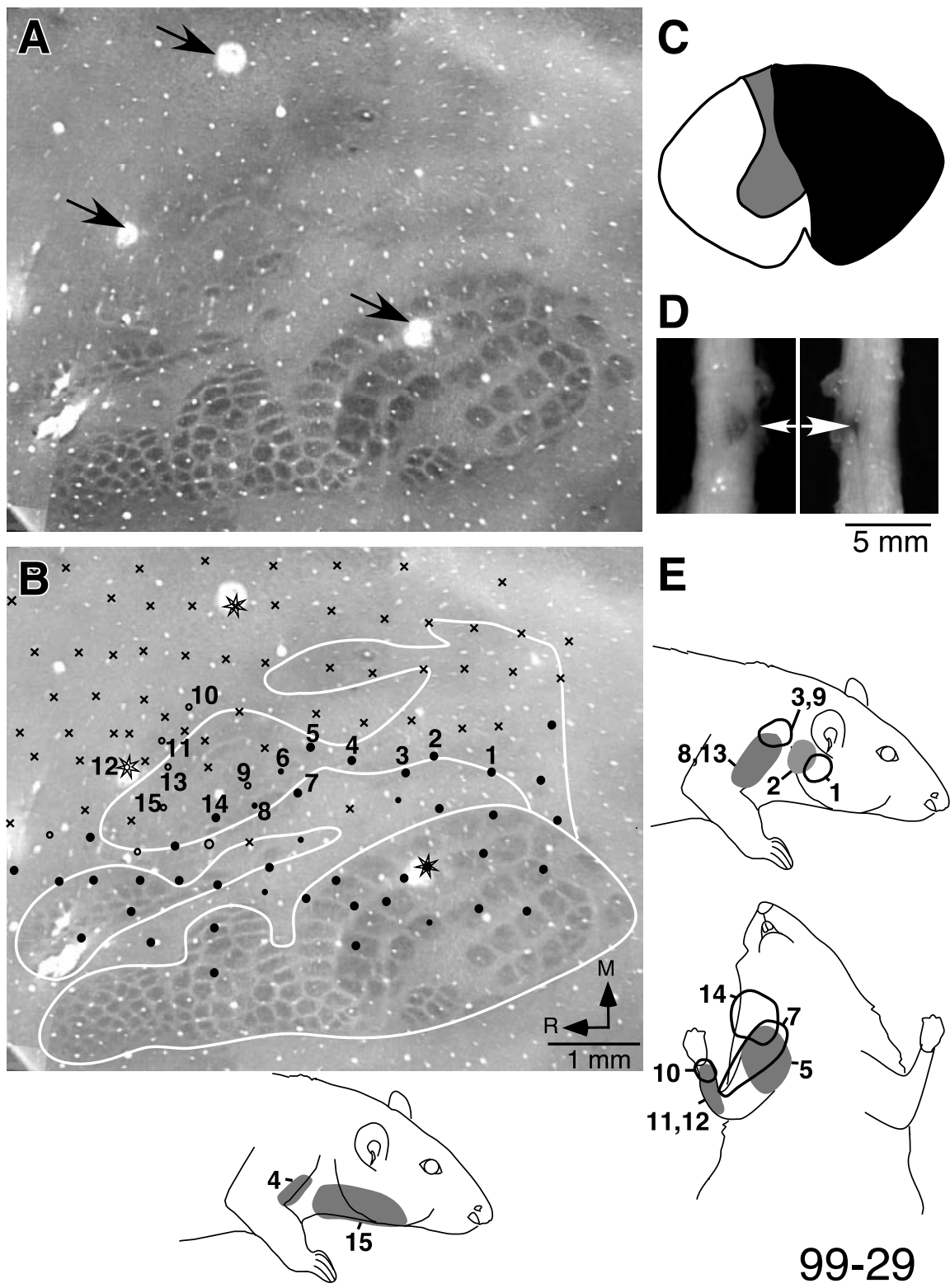

E
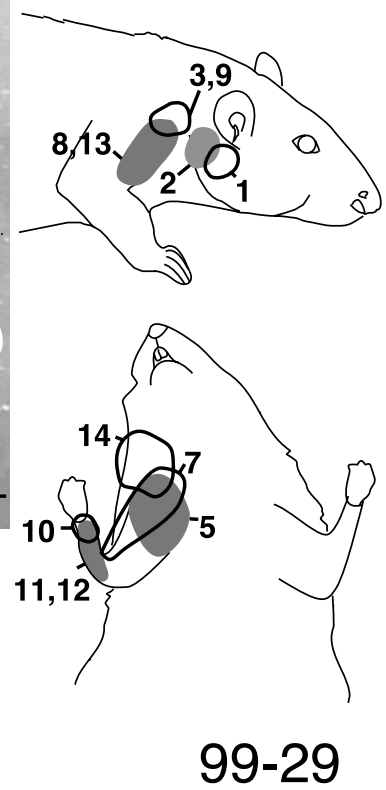

Figure 2. Changes in the modular pattern and somatotopy in $S 1$ as a result of neonatal spinal overhemisection in rat 99-29. $A$, The modular pattern in the $S 1$ cortex revealed by staining sections of the flattened cortex for $C 0$ activity. Note that the modules are absent in the forepaw and hindpaw regions, although they are normal in the face region. In the forepaw region, only a few faint modules in the lateralmost and medialmost regions are discernable. $B$, Electrode penetration sites and responsiveness in $S 1$ cortex. Neuronal responses in the region of the face and lower lip representations remain normal. The neurons respond vigorously to light touch (large filled circles) on the whiskers and hairs (compare with Fig. 1). In the regions of the forepaw and hindpaw at most of the sites, the neurons were unresponsive ( $X$ symbols) or responded weakly to cutaneous (small filled circles) or deep (small open circles) stimulation. At these responsive sites, the receptive fields of neurons are located on the skin of the arm, shoulder, or neck, the inputs that enter the spinal cord rostral to the lesion (see $E$ ). There are no responses in the forepaw region of the $\mathrm{S} 1$ cortex to the stimulation of the forepaw. Electrolytic microlesions made to help overlay the histochemically visible map in $\mathrm{CO}$-stained sections with the electrophysiological map are marked with arrows in $A$ and $\operatorname{stars}$ in $B$. The expected normal outline of the body representation (white outline in $B$ ) is approximated based on the face representation for this rat and the body representation in a normal rat (see Fig. 1). R, Rostral; M, medial. C, Reconstruction of the spinal cord lesion site in a coronal plane showing the extent of the lesion. The damaged portion is shown in black. Note that the overhemisection is complete. D, Photographs of the spinal cord showing the lesion (arrows) in a dorsal (left) and a ventral (right) view. $E$, Receptive fields at selected numbered locations in the forepaw region of $\$ 1$.

from one rat (Fig. 4, rat 99-35) were processed for Nissl substance, and those from one rat (Figs. 5, 6, rat 99-43) were processed for myelin. In three rats, 99-29, 99-33, and 99-27, the CO-stained sections of the cortex showed that the modules were absent in the regions of the forepaw representation, except for a 

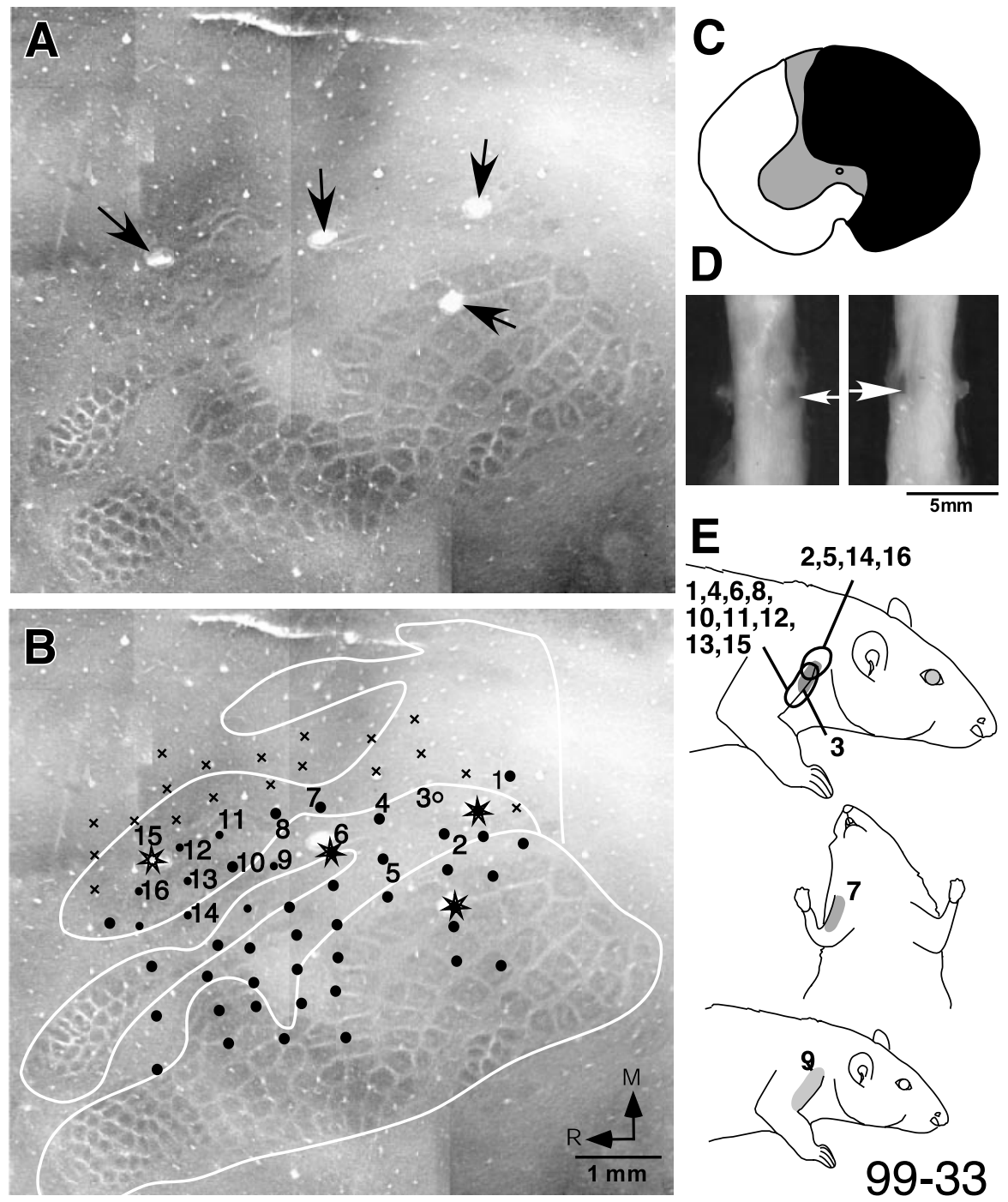

Figure 3. Changes in the modular pattern and somatotopy in $\mathrm{S} 1$ as a result of neonatal spinal overhemisection in rat 99-33. $A$, The modular pattern in the $\mathrm{S} 1$ cortex revealed by staining sections of the flattened cortex for $\mathrm{C} O$ activity. Note that the modules are absent in the forepaw and hindpaw regions, although they are normal in the face region. In the forepaw region only, a few faint modules in the lateralmost and medialmost regions are discernable. $B$, Electrode penetration sites and responsiveness in S1 cortex. Neuronal responses in the region of the face and lower lip representations remain normal. The neurons respond vigorously to light touch (large filled circles) on the whiskers and hairs (compare with Fig. 1). In the region of the forepaw at many of the sites, the neurons were unresponsive $(X$ symbols) or responded weakly to cutaneous (small filled circles) stimulation. At these responsive sites, the receptive fields of neurons are located on the skin of the arm or shoulder, the inputs that enter the spinal cord rostral to the lesion (see $E$ ). There are no responses in the forepaw region of the $S 1$ cortex to the stimulation of the forepaw. Electrolytic microlesions made to help overlay the histochemically visible map in CO-stained sections with the electrophysiological map are marked with arrows in $A$ and stars in $B$. The expected normal outline of the body representation (white outline in $B$ ) is approximated based on the face representation for this rat and the body representation in a normal rat (see Fig. 1). $R$, Rostral; M, medial. C, Reconstruction of the spinal cord lesion site in a coronal plane showing the extent of the lesion. Note that the overhemisection is complete except for small remaining fibers in the ventromedial region. D, Photographs of the spinal cord showing the lesion (arrows) in a dorsal (left) and a ventral (right) view. E, Receptive fields at selected numbered locations in the forepaw region of $S 1$.

few faint bands in the medialmost and lateralmost edges (Figs. $2 A, 3 A$ ). The intensity of the $\mathrm{CO}$ staining in the deprived forepaw region appears only slightly higher than the surrounding cortex and much lighter than for barrels in the PMBSF region. Thus, the deprived region of $\mathrm{S} 1$ lacked modular subdivisions, and the metabolic activity was reduced. In one of the rats (99-41), the CO patches were faintly apparent in the forepaw region, although the patches did not resemble the normal pattern. The presence of patches in this rat could be attributable to the presence of a few intact fibers that could be present in the scar tissue and were not discernible in the histological sections of the spinal cord (Jain et al., 1995, 1997), but such inputs were not detected in recordings from cortex. However, some neurons in the hindpaw region responded to the stimulation of the hindpaw (see below). In the S1 cortex stained for Nissl substance (Fig. 4A, rat 99-35), there were no modular arrangements of the cells in the forepaw region. Instead, there was a narrow uniformly cell-dense region in the expected location of the forepaw representation. The cell-dense regions do however indicate that at least part of the deafferented S1 cortex retains a granular appearance. Finally, the sections of the flattened cortex from rat 99-43 were stained for myelin. The staining pattern was uniformly diffuse in the forepaw region, without any evidence of patches or the myelin-dark septa in layer 4 or the myelin-dark patches in the deeper layers of cortex (Figs. 5A, 6, rat 9943). The modular pattern was disrupted in the hemisphere ipsilateral to the hemisection as well. The use of these three different histological techniques to reveal the presence of barrels and patches in S1 shows that the neuronal organization in the cortex, levels of the metabolic activity in the dendrites and thalamocortical axon terminals (Wong-Riley and Welt, 1980), and the arrangement of thalamocortical afferents all are disrupted as a result of the spinal lesions.

We did not observe any obvious increase in the size of the PMBSF compared with the normal animals. In a similar manner, neither forepaw amputation (Killackey and Dawson, 1989) nor infraorbital nerve section (Killackey et al., 1994) increased the size of the intact representations unless the deafferentation was done in embryonic stages.

\section{Lack of responses to the deafferented parts of the body in $S 1$ and plasticity} We recorded neuronal activity from the left $S 1$ contralateral to the hemisection side in an effort to determine the role of isolated spinothalamic inputs in the development of the somatosensory system. This side did not receive inputs via the uncrossed dorsal quadrant, but the crossed spinothalamic inputs were intact. In normal rats, the receptive fields of neurons in any part of S1 precisely match the somatotopy revealed by various histological markers. The region of S1 in which neurons respond to the stimulation of the skin of the forepaw lies just medial to the representation of the lower lip, approximately parallel to the representation of nose and rostral vibrissae and the furry buccal pad. Caudal to the representation of the forepaw, responses to the stimulation of the wrist, forearm, and upper arm are observed (Welker, 1976; Chapin and Lin, 1984; Waters et al., 1995) (Fig. 1). 
We did not encounter any responses to the stimulation of the forepaw in any of the deafferented rats. In large parts of $\mathrm{S} 1$ in which the representation of the forepaw is expected, neurons did not respond to the stimulation of either the cutaneous or the deep receptors of any part of the body. In all of the rats, neurons in the caudal regions of the forelimb representation responded to the stimulation of the upper arm and shoulder, the region in which these representations are normally located. Inputs from these parts of the body enter the spinal cord rostral to the site of the lesion. In addition to activating neurons in their normal locations, these inputs also activated neurons in variable extents of the deafferented forepaw region. For example, in rat 99-43, such neurons were restricted to the caudalmost region of the forelimb representation (Fig. $5 B, D$, see receptive fields at locations 1-8), whereas in rat 99-35, the upper armshoulder responsive zone expanded into large parts of the forepaw region (Fig. $4 B, D$, see receptive fields at locations $1-6$, $10-16,18,20)$. The expanded representation of these intact inputs for the remaining rats was variable between these two extremes. The responses in these zones of expanded representations were either weak or required taps instead of a light cutaneous touch or movement of hairs.

In addition to the expansion of the upper arm-shoulder representations, we also noted a few sites in the forepaw region of $\mathrm{S} 1$ in which neurons responded weakly to the stimulation of the neck. These few sites were primarily located in the lateralmost regions, closer to the normal neck representation (Fig. $2 B, E$, see receptive fields at locations 14,15$)$.

Thus, in the lesioned rats, representations of the upper arm and chest show limited expansion into parts of the deafferented forepaw region. There was also a limited medial expansion of the neck representation, which is normally represented toward the caudal region of the lower jaw representation (Chapin and Lin, 1984).

In three rats (99-29, 99-41, and 99-43), we also mapped the medial regions of $\mathrm{S} 1$ that correspond to the region of the foot representation. In two of the rats (Figs. $2 B, 5 B$, rats 99-29 and 99-43) with complete hemisections, there were no responses to the stimulation of any part of the body in this region. In one of the rats (99-41), we observed responses to the stimulation of leg, foot, and toes. However, the order of the receptive fields did not show any particular somatotopic order, indicating that the partially intact hindlimb inputs activated both normal and abnormal locations within the region of the normal hindlimb and foot representations.

In all of the rats, responses to the stimulation of the whiskers, upper lip, and lower lip were normal and were at the expected
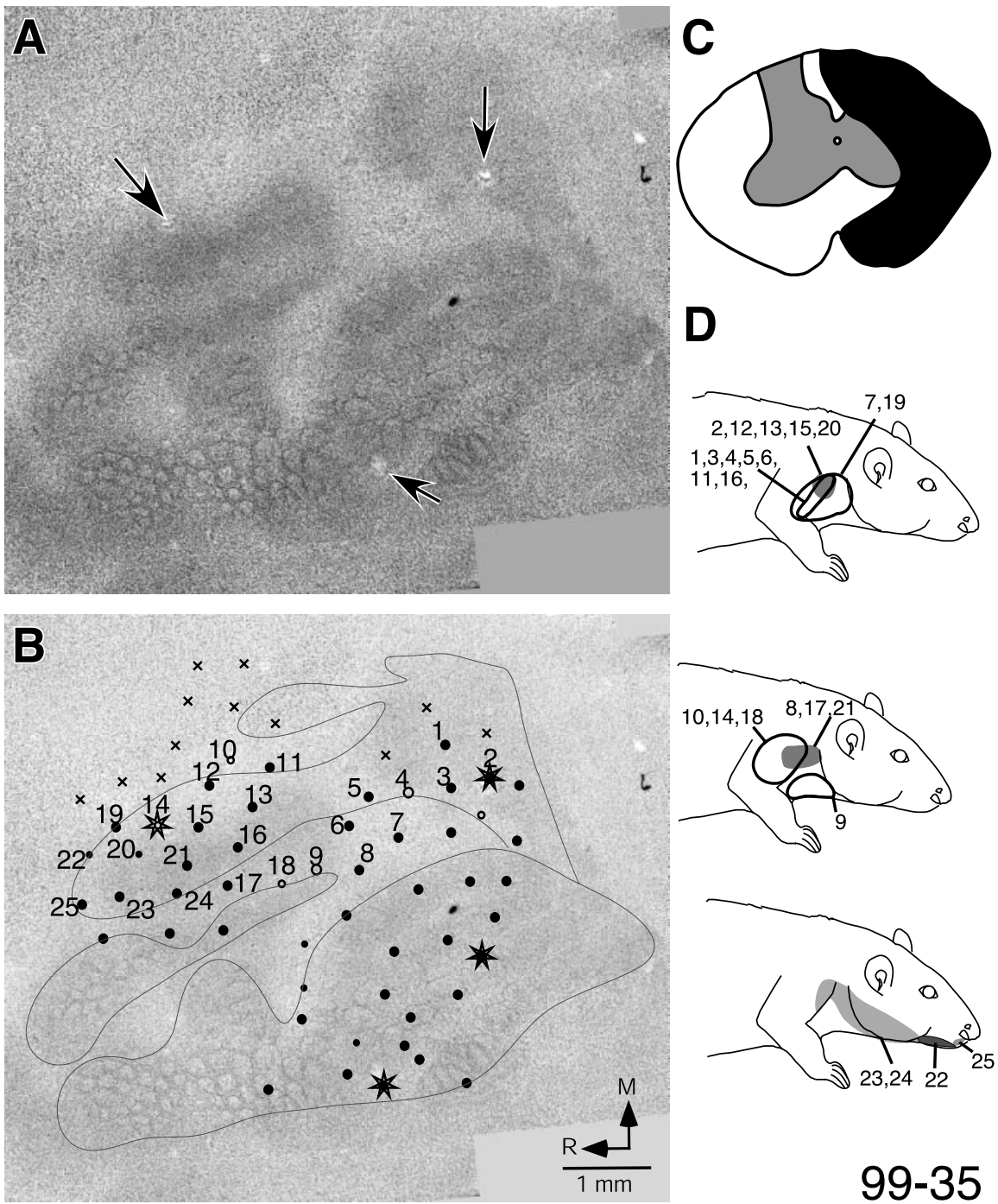

99-35

Figure 4. Changes in the modular pattern and somatotopy in $\mathrm{S} 1$ as a result of neonatal spinal overhemisection in rat 99-35. A, The modular pattern in the $\mathrm{S1}$ cortex revealed by staining sections of the flattened cortex for Nissl substance. Note that the modules are absent in the forepaw and hindpaw regions, although they are normal in the face region. The forepaw and the hindpaw regions have small uniform cell-dense regions devoid of any modules (compare with Fig. $1 B$ ). $B$, Electrode penetration sites and responsiveness in $\mathrm{S} 1$ cortex. Neuronal responses in the region of the face and lower lip representations remain normal. The neurons respond vigorously to light touch (large filled circles) on the whiskers and hairs (compare with Fig. 1). In the regions of the forepaw and hindpaw at most of the sites, the neurons were unresponsive ( $\times$ symbols) or responded to cutaneous stimulation (filled circles). At these responsive sites, the receptive fields of neurons are located on the skin of the arm, shoulder, neck, or lower lip, the inputs that enter the spinal cord rostral to the lesion (see $D$ ). There are no responses in the forepaw region of the $\mathrm{S} 1$ cortex to the stimulation of the forepaw. Electrolytic microlesions made to help overlay the histochemically visible map in the Nissl-stained sections with the electrophysiological map are marked with arrows in $A$ and stars in $B$. The expected normal representation in a normal rat (see Fig. 1). R, Rostral; M, medial. C, Reconstruction of the spinal cord lesion site in a coronal plane showing the extent of the lesion. $D$, Receptive fields at selected numbered locations in the forepaw region of $S 1$ cortex.

locations, matching the isomorphs of these regions revealed in the section of the flattened cortices (Figs. 2-5).

\section{Anatomical evidence for preserved afferents from the arm}

To determine whether the observed responses to the stimulation of the upper arm were indeed from the intact inputs entering the spinal cord rostral to the site of the lesion, we injected B-HRP in the skin of the forearm and upper arm of three rats (99-35, 99-41, and 99-43). If all the dorsal column inputs to the cuneate nucleus, including those from the upper arm, were severed, we would expect to see no label in the cuneate nucleus. However, in all of these rats, we observed TMB reaction product in the dorsomedial 


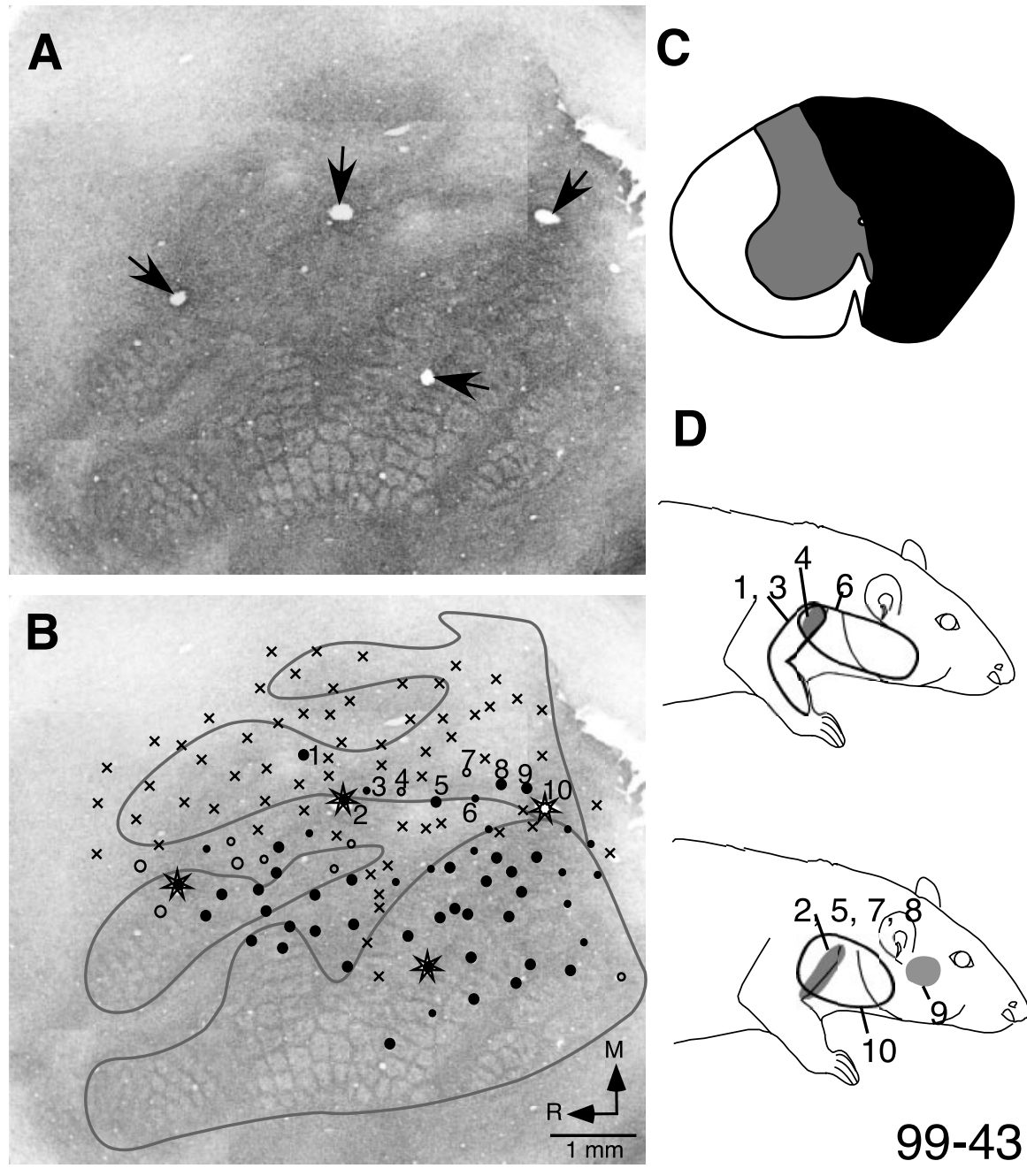

Figure 5. Changes in the modular pattern and somatotopy in $\mathrm{S} 1$ as a result of neonatal spinal overhemisection in rat 99-43.A, The modular pattern in the $S 1$ cortex revealed by staining sections of the flattened cortex for myelin. Note that the modules are absent in the forepaw and hindpaw regions, although they are normal in the face region. The forepaw and the hindpaw regions show dark uniform staining as for the trunk region (compare with Fig. 1D; also see Fig. 6). B, Electrode penetration sites and responsiveness in $\mathrm{S} 1$ cortex. Neuronal responses in the region of the face and lower lip representations remain normal. The neurons respond vigorously to light touch (large filled circles) on the whiskers and hairs (compare with Fig. 1). In the regions of the forepaw and hindpaw at nearly all of the sites, the neurons were unresponsive $(X$ symbols). At a few sites, the neurons responded to cutaneous (filled circles) or deep (small open circles) stimulation. At these responsive sites, the receptive fields of neurons are located on the skin of the arm, shoulder, or neck, the inputs that enter the spinal cord rostral to the lesion (see $D$ ). There are no responses in the forepaw region of the $S 1$ cortex to the stimulation of the forepaw. Electrolytic microlesions made to help overlay the histochemically visible map in the myelin-stained sections with the electrophysiological map are marked with arrows in $A$ and stars in $B$. The expected normal outline of the body representation (dark outline in $B$ ) is approximated based on the face representation for this rat and the body representation in a normal rat (see Fig. 1). R, Rostral; M, medial. C, Reconstruction of the spinal cord lesion site in a coronal plane showing the extent of the lesion. $D$, Receptive fields at selected numbered locations in the forepaw region of $\mathrm{S1}$ cortex.

region of the cuneate nucleus (Fig. 7), the region in which inputs from the arm normally terminate (Maslany et al., 1990; Xu and Wall, 1999), indicating that the responses to the stimulation of the upper arm in the forepaw and arm regions of S1 were attributable to the preservation and expansion of the intact upper arm inputs that enter the spinal cord rostral to the site of the lesion. This was further demonstrated in the sections of the spinal cord, which clearly showed the presence of the tracer in the dorsal columns rostral to the lesion.

\section{Effect of spinal hemisections on the brainstem nuclei}

We stained sections from the lower medulla for cytochrome oxidase activity to reveal changes in the organization of the brain- stem nuclei. In normal rats (Fig. 8), the cuneate nucleus, particularly in the central pars rotunda region, consists of clusters of large neurons, which stain densely for $\mathrm{CO}$ activity. The clusters are surrounded by CO-light regions. The dorsal column afferents from the forepaw primarily terminate in these CO-dark clusters (Crockett et al., 1993). In rats with neonatal spinal hemisections, no CO-dark clusters were apparent in the deprived cuneate nucleus. In addition, the cuneate nucleus and the gracile nucleus showed a variable reduction in size depending on the extent of the lesion.

\section{Discussion}

We found that the normal histologically visible pattern of modules is absent in the deprived regions of $\mathrm{S} 1$ cortex after partial loss of ascending somatosensory inputs resulting from neonatal cervical spinal cord overhemisections. In cortex deprived of dorsal quadrant inputs, neurons in the region of the expected forepaw representation did not respond to the stimulation of the deafferented parts of the body. Although large parts of the deafferented cortex remain unresponsive, intact inputs from the upper arm that enter the spinal cord rostral to the site of the lesion activated an expanded zone of cortex that included some of the deafferented forepaw cortex. These results support three major conclusions about the development and plasticity of the somatosensory system. (1) The crossed spinothalamic inputs in the ventral quadrant are unable to sustain the formation of a normal neuroanatomical map in the rat $\mathrm{S} 1$. For the formation of the normal modular pattern, uncrossed dorsal quadrant inputs are essential. (2) The spinothalamic inputs do not become capable of activating neurons in the somatosensory cortex, even when the dorsal quadrant inputs are removed early in development. (3) Whereas the few intact afferents from the upper arm in the dorsal columns come to activate more than their normal territory in S1, the surviving trigeminal afferents from the face do not activate a notably larger than normal territory. Thus, the developing system responds to surviving face and arm afferents differently.

\section{Effects of deafferentations on the formation of the barrel pattern in the primary somatosensory cortex}

In the rat somatosensory system, the development of the forelimb pathway lags the whisker-to-S1 cortex pathway by $\sim 1 \mathrm{~d}$ and precedes the development of the hindlimb pathway by $1 \mathrm{~d}$. Barrels are detectable soon after the appearance of thalamocortical afferents even before layer IV is generated (Erzurumlu and Jhaveri, 1990). Acetylcholine histochemistry reveals that the thalamocortical afferents segregate into barrel-like patches by late P1 for the 


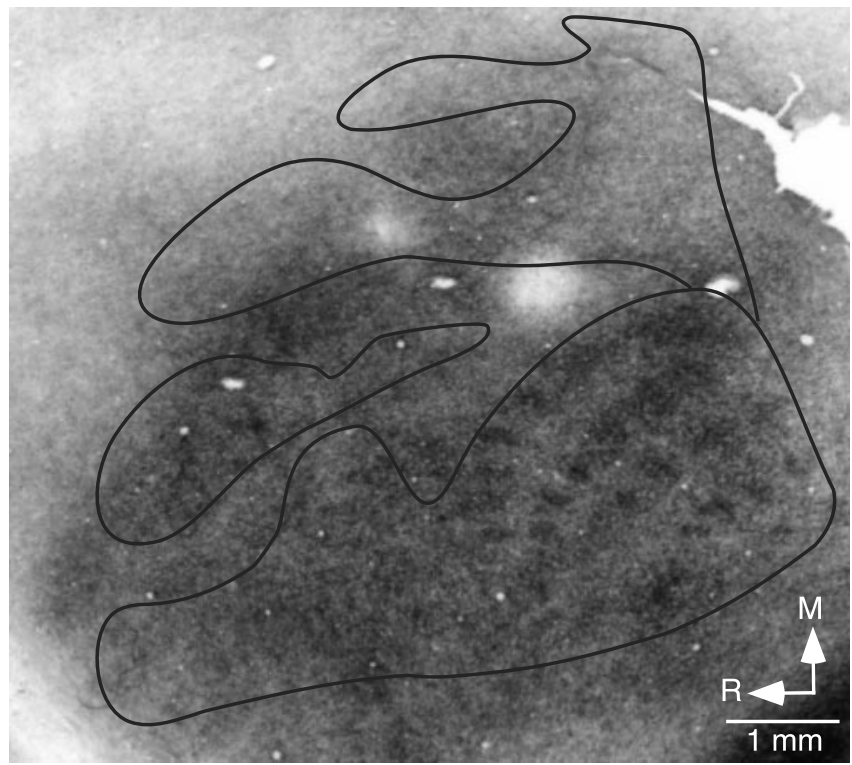

Figure 6. Changes in the modular pattern and somatotopy in $\$ 1$ as a result of neonatal spinal overhemisection in rat 99-43. The modular pattern in the deeper layers of $\$ 1$ cortex revealed by staining sections of the flattened cortex for myelin. Note that the modules show a pattern that is reverse of that seen in the middle layers (compare with Figs. 1D, 5A). In deeper layers, the barrels are myelin dark surrounded by myelin-light septa. The modules are absent in the forepaw and hindpaw regions, although they are normal in the face region. The modules are not clearly visible in the rostral face representation because this image is from the photomicrograph of a single section, and part of the modules were in other sections attributable to uneven flattening. (The image shown in Fig. $5 \mathrm{~A}$ is a photomontage.)

whisker barrels and by P3 for the forepaw barrels (Schlagger and O'Leary, 1994; the day of birth is $\mathrm{P} 1$, and, in reviewing the previous literature, we have adjusted accordingly). In Nissl and succinic dehydrogenase preparations, the barrels become visible after an additional 2 d (Killackey and Belford, 1979; Rice et al., 1985). The critical period for the development of the forepaw modules as well as the whisker barrels ends at approximately P6, well after we did our lesions.

Previously, it has been shown that complete deafferentation by removal of the forelimb on $\mathrm{P} 3$ or $\mathrm{P} 4$ results in a complete disruption of the modules in the forepaw region of the S1 cortex (Waters et al., 1990; Pearson et al., 1999). Our results show that transecting uncrossed pathways in the dorsal and dorsolateral spinal cord, although leaving the crossed spinothalamic pathways intact, is sufficient to disrupt the formation of the normal pattern of modules in the forepaw and hindpaw regions of the S1 cortex. Spinothalamic afferents, which remained intact in our preparation, terminate throughout the ventroposterior nucleus of the thalamus intermingled with terminations of lemniscal inputs (Ma et al., 1986). In rats, the synapses of both of these ascending systems appear to be similar and indistinguishable (Peschanski et al., 1985), and $\sim 10 \%$ of the spinothalamic terminations are on the neurons that also receive lemniscal inputs (Ma et al., 1987). However, it is possible that cutaneous inputs via the upper quadrant of the spinal cord provide the most patterned activity to the developing system, whereas the crossed spinothalamic inputs with mostly large overlapping receptive fields (Willis and Coggeshall, 1991; Dado et al., 1994) are not sufficient to direct the maintenance of segregated thalamocortical afferents and the development of the modules. Alternatively, the level of subthreshold activation via spinothalamic inputs, if any, may not be sufficient to overcome the loss of patterned activity attributable to the

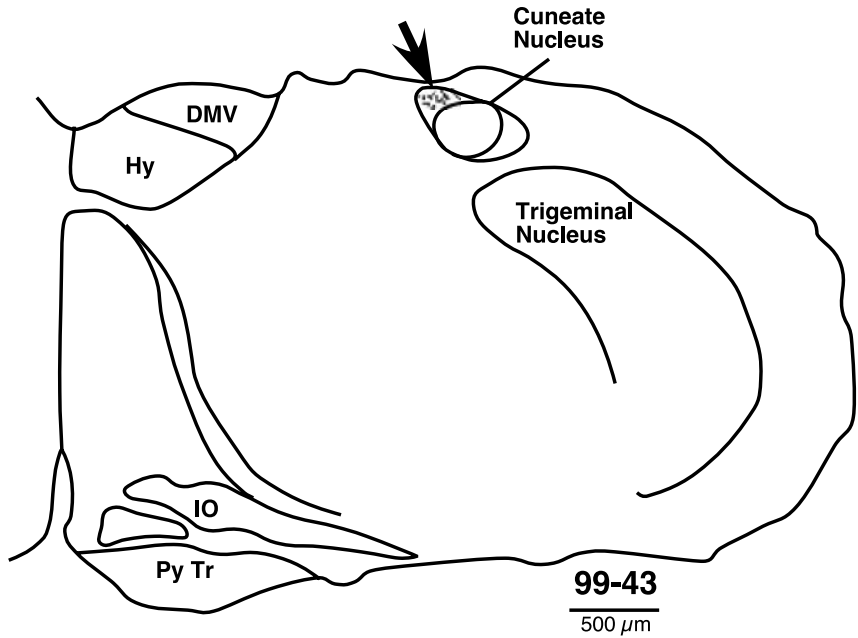

Figure 7. Plot of the transganglionic neuronal tracer B-HRP in a section from the lower medulla of rat $99-43$. The tracer was injected in the skin of the upper arm (between the shoulder and the elbow) at 13 different locations. Presence of the tracer in the brainstem shows that dorsal column inputs from this part of the arm were not interrupted by the spinal lesion because they entered the spinal cord rostral to the lesion. DMV, Dorsal motor nucleus of the vagus nerve; Hy, hypoglossal nucleus; 10, inferior olivary nucleus; Py Tr, pyramidal tract.

spinal hemisections. We see no evidence in anesthetized rats of neural spikes in $\mathrm{S} 1$ that are generated by spinothalamic pathways. In support of the present results, lesions of the principal trigeminal nucleus, the major target of the trigeminal analog of the dorsal column afferents, are sufficient to disrupt the barreloid pattern in the ventroposterior nucleus of the thalamus (Killackey and Fleming, 1985). An intact spinal trigeminal nucleus with normal barrel patterns (barrelettes) in the subnucleus interpolaris and caudalis of the spinal trigeminal nucleus cannot sustain the formation of a normal thalamic barreloid pattern (Killackey and Fleming, 1985).

\section{Somatotopic reorganization}

Although a large number of studies have examined the effects of embryonic or neonatal peripheral injuries on the barrel pattern as revealed by various histochemical stains, only a few examined the changes in topography to determine correspondence between the altered barrel pattern and the receptive field properties of neurons. In general, it appears that deafferentations done early in development do not lead to a large-scale expansion of the intact, topographically adjacent body regions into the deafferented cortex. Rhoades and colleagues (Lane et al., 1995) report that, after forelimb amputation on P1 (the day of birth), neurons at most of the recording sites in the forepaw region were responsive to the stimulation of the stump, and only at a few sites were there responses to the stimulation of the hindlimb or inputs from the chin, whiskers, or neck. In a similar study, Pearson et al. (1999) found that amputation of the forelimb on P3 leads to multiple islands of stump-responsive sites in the forelimb area. The expansion of the trigeminal inputs into the forelimb area was extremely limited and confined to the region adjacent to the face representation. Even the forelimb amputations done earlier, on embryonic day 16 or 17 , failed to produce responses to the stimulation of the intact hindlimb in the deafferented forelimb region of S1 (Killackey and Dawson, 1989).

The lack of activation by the spinothalamic inputs and the lack of any extensive large-scale reorganization after early deafferentations reported here is similar to that found after lesions in adult 
A
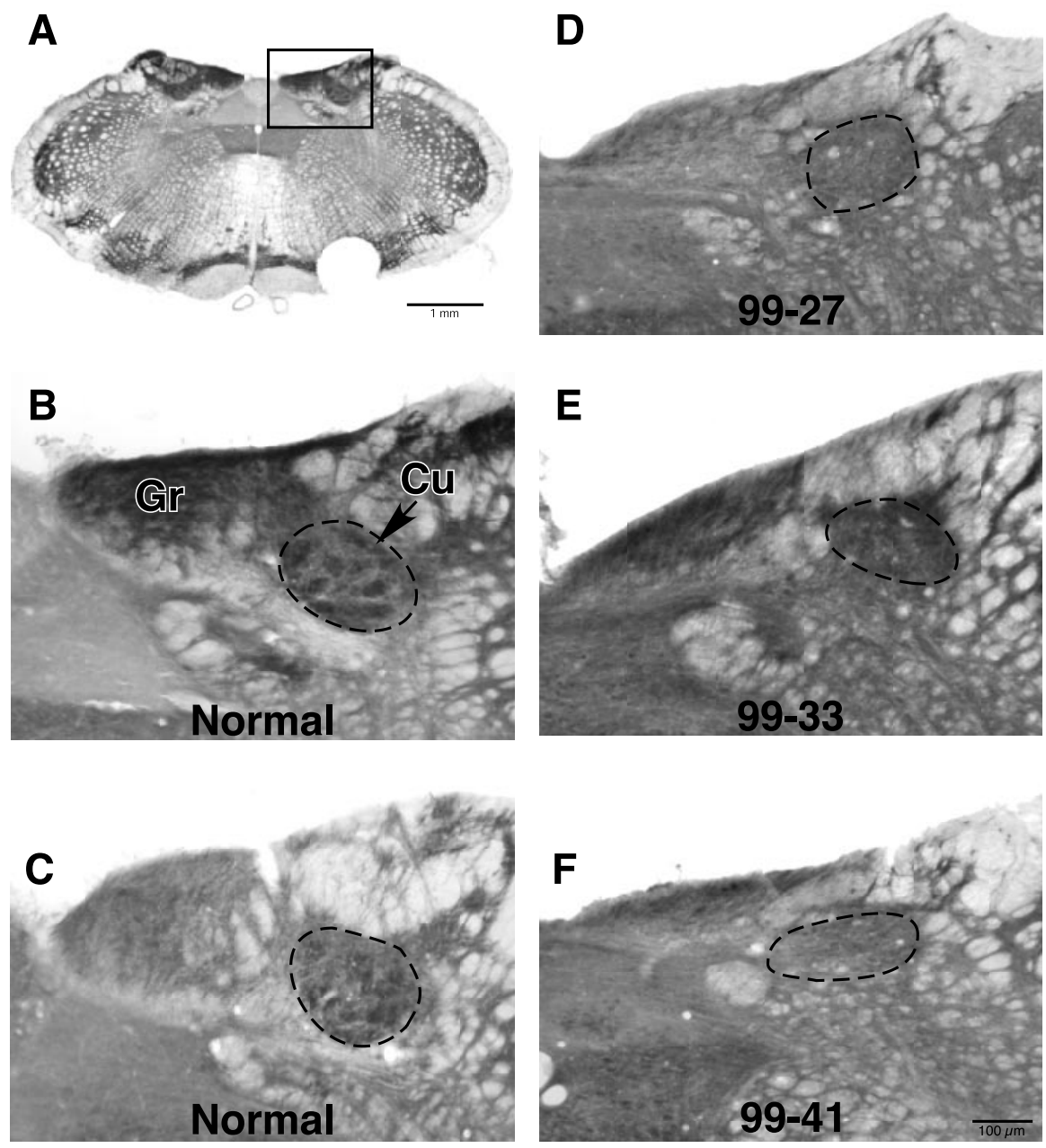

Figure 8. Photomicrographs of sections through the lower medulla stained for cytochrome oxidase activity. $A, A$ complete section through the medulla of a normal rat. The region shown at a higher magnification in the other panels is boxed. $B, C$, High-magnification photomicrographs from lower medulla in the region of the cuneate nucleus (Cu) from two normal rats. Note the $\mathrm{C} 0$-dark clusters in the pars rotunda of the cuneate nucleus (outlined with dashed line) in which dorsal column inputs terminate. $D-F$, Photomicrographs of the brainstem in the region of the cuneate nucleus at approximately the same rostrocaudal plane as for $B$ and ( from three rats, 99-27, 99-33, and 99-41, that had undergone neonatal spinal overhemisections. Note that the pars rotunda of the cuneate nucleus (dashed outline) has a uniform appearance lacking $C 0$-dark clusters. Gr, Nucleus gracilis. Scale bar shown in $F$ also applies to $B-E$.

rats. Lesions of the dorsal columns of the spinal cord at thoracic levels in adult rats do not lead to an expansion of the adjacent intact forelimb representation into the deafferented hindlimb areas of S1 (Jain et al., 1995) (but see Wall and Egger, 1971). The results from the early-lesioned rats are also similar to those in early-lesioned primates in which reactivation of the deafferented cortex by the intact face inputs after dorsal column injuries is rather limited (Jain et al., 2001b). However, the lack of expansion of the intact inputs in rats lesioned as adults (Jain et al., 1995) is different from the primate somatosensory system in which such lesions lead to an extensive expansion of the trigeminal inputs into the deafferented cortex (Jain et al., 1997, 2000a). The mechanisms underlying these age-dependent differences between rodents and primates are presently not clear. Lesions of the spinal cord in newborn cats lead to extensive reorganization in the S1 cortex, including duplication of the representations of the remaining inputs (McKinley et al., 1987). However, these duplications were observed after complete transection of the spinal cord, and it is possible that the intact spinothalamic inputs in the ex- periments in rats and monkeys were able to prevent such large rearrangements in the topographic maps.

Possible mechanisms of the topographic reorganization and the subcortical changes

In normal animals, corticocortical connections between the shoulder and the forepaw regions of $\mathrm{S} 1$ are absent (Pearson et al., 1999) (but see Fabri and Burton, 1991). Because expansion of the stump or other representations in the cortex after limb amputation at P1 or P3 (Stojic et al., 1998; Pearson et al., 1999) is not accompanied by appearance of any new corticocortical connections between the hindlimb-forelimb or shoulder-forepaw regions, even the limited cortical reorganization is likely to be a reflection of the subcortical changes rather than intracortical mechanisms.

The brainstem nuclei are extensively disrupted after early deafferentations. The changes include absence of the CO-dark patches (Killackey and Dawson, 1989; present results), shrinkage of the nucleus (Rhoades et al., 1993), and apparent fusion of the gracilis and cuneate nuclei (Killackey and Dawson, 1989) (but see Rhoades et al., 1993). There is also growth of hindlimb afferents from the gracile nucleus into the cuneate nucleus after forelimb amputation (Rhoades et al., 1993; Lane et al., 1995). In these animals, there is no growth from the trigeminal nucleus, apparently because the trigeminal pathway develops before the forelimb pathway. In our lesions, disruption of afferents to both the cuneate and the gracile nucleus precludes such growth.

Despite the lack of neuronal responsiveness in S1 cortex, it is surprising that many of these rats develop forelimb reaching, rear on their hindlimbs, and walk overground. Forelimb, trunk, and hindlimb motor skills typically seen in the adult rat develop well when rats are exposed to enriched environment. Skilled forelimb movements include reaching for targets placed both on and away from the body, a task requiring refined postural adjustments, including rapid repositioning of hindlimbs and feet. In addition, appropriate postural adjustments accompany the forelimb reaching to minimize falls or loss of balance during forelimb activities (Diener, 2002). Perhaps connections between supraspinal and segmental-intersegmental pathways (Diener, 2002) in conjunction with ventral quadrant sensory pathways are sufficient for the recovery of reaching skills and compensatory postural adjustments, even in the absence of fine-grained patterned activation of the $\mathrm{S} 1$ cortex.

\section{References}

Chapin JK, Lin C-S (1984) Mapping the body representation in the SI cortex of anaesthetized and awake animals. J Comp Neurol 229:199-213.

Crockett DP, Maslany S, Harris SL, Egger MD (1993) Enhanced 
cytochrome-oxidase staining of the cuneate nucleus in the rat reveals a modifiable somatotopic map. Brain Res 612:41-55.

Dado RJ, Katter JT, Giesler Jr GJ (1994) Spinothalamic and spinohypothalamic tract neurons in the cervical enlargement of rats. II. Responses to innocuous and noxious mechanical and thermal stimuli. J Neurophysiol 71:981-1002.

Dawson DR, Killackey HP (1987) The organization and mutability of the forepaw and hind paw representations in the somatosensory cortex of neonatal rat. J Comp Neurol 256:246-256.

Diener P (2002) Enriched environments improve skilled movements following neonatal cervical spinal cord injury in rats. Soc Neurosci Abstr 28:633.620.

Erzurumlu RS, Jhaveri S (1990) Thalamic axons confer a blueprint of the sensory periphery onto the developing rat somatosensory cortex. Dev Brain Res 56:229-234.

Fabri M, Burton H (1991) Ipsilateral cortical connections of primary somatic sensory cortex in rats. J Comp Neurol 311:405-424.

Florence SL, Kaas JH (1995) Large-scale reorganization at multiple levels of the somatosensory pathway follows therapeutic amputation of the hand in monkeys. J Neurosci 15:8083-8095.

Gibson AR, Hansama DI, Robinson FR (1984) A Sensitive low artifact TMB procedure for the demonstration of WGA-HRP in the CNS. Brain Res 298:235-241.

Jain N, Florence SL, Kaas JH (1995) Limits on plasticity in somatosensory cortex of adult rats: hindlimb cortex is not reactivated after dorsal column section. J Neurophysiol 73:1537-1546.

Jain N, Catania KC, Kaas JH (1997) Deactivation and reactivation of somatosensory cortex after dorsal spinal cord injury. Nature 386:495-498.

Jain N, Catania KC, Kaas JH (1998) A histologically visible representation of the fingers and palm in primate area $3 \mathrm{~b}$ and its immutability following long term deafferentation. Cereb Cortex 8:227-236.

Jain N, Florence SL, Qi H-X, Kaas JH (2000a) Growth of new brain stem connections in adult monkeys with massive sensory loss. Proc Natl Acad Sci USA 97:5546-5550.

Jain N, Coq J-O, Diener PS, Kaas JH (2000b) Physiological and anatomical reorganization of the somatosensory cortex after spinal hemisections in neonatal rats. Somatosens Mot Res 17:93.

Jain N, Qi H-X, Catania KC, Kaas JH (2001a) Anatomical correlates of the face and oral cavity representation in somatosensory area $3 \mathrm{~b}$ of monkeys. J Comp Neurol 429:455-468.

Jain N, Qi H-X, Collins CE, Lyon DL, Kaas JH (2001b) Reorganization of somatosensory cortical area $3 \mathrm{~b}$ following early postnatal dorsal column lesions in macaque monkeys. Soc Neurosci Abstr 27:396.1.

Jensen KF, Killackey HP (1987) Terminal arbors of axons projecting to the somatosensory cortex of the adult rat. II. The altered morphology of thalamocortical afferents following neonatal infraorbital nerve cut. J Neurosci 7:3544-3553

Killackey HP, Belford GR (1979) The formation of afferent patterns in the somatosensory cortex of the neonatal rat. J Comp Neurol 183:285-303.

Killackey HP, Dawson DR (1989) Expansion of the central hindpaw representation following fetal-forelimb removal in rats. Eur J Neurosci $1: 210-221$

Killackey HP, Fleming K (1985) The role of the principal sensory nucleus in central trigeminal pattern formation. Brain Res 354:141-145.

Killackey HP, Chiaia NL, Bennett-Clarke CA, Eck M, Rhoades RW (1994) Peripheral influences on the size and organization of somatotopic representations in the fetal rat cortex. J Neurosci 14:1496-1506.

Lane RD, Bennett-Clarke CA, Chiaia NL, Killackey HP, Rhoades RW (1995) Lesion-induced reorganization in the brainstem is not completely expressed in somatosensory cortex. Proc Natl Acad Sci USA 92:4264-4268.

Ma W, Peschanski M, Bessen JM (1986) The overlap of spinothalamic and dorsal column nuclei projections in the ventrobasal complex of the rat thalamus: a double anterograde labeling study using light microscopy analysis. J Comp Neurol 245:531-540.

Ma W, Peschanski M, Ralston HJ (1987) The differential synaptic organization of the spinal and lemniscal projections to the ventrobasal complex of the rat thalamus. Evidence for convergence of the two systems upon single thalamic neurons. Neuroscience 22:925-934.

Maslany S, Crockett DP, Egger MD (1990) Somatotopic organization of the cuneate nucleus in the rat: transganglionic labelling with WGA-HRP. Brain Res 507:164-167.

McKinley PA, Jenkins WM, Smith JL, Merzenich MM (1987) Agedependent capacity for somatosensory cortex reorganization in chronic spinal cats. Brain Res 428:136-139.

Pearson PP, Li CX, Waters RS (1999) Effects of large-scale limb deafferentation on the morphological and physiological organization of the forepaw barrel subfield (FBS) in somatosensory cortex (SI) in adult and neonatal rats. Exp Brain Res 128:315-331.

Peschanski M, Roudier F, Ralston III HJ, Besson J-M (1985) Ultrastructural analysis of the terminals of various somatosensory pathways in the ventrobasal complex of rat thalamus: an electron microscopic study using wheatgerm agglutinin conjugated to horseradish peroxidase as an axonal tracer. Somatosen Res 3:75-87.

Rhoades RW, Wall JT, Chiaia NL, Bennett-Clarke CA, Killackey HP (1993) Anatomical and functional changes in the organization of the cuneate nucleus of adult rats after fetal forelimb amputation. J Neurosci 13:1106-1119.

Rhoades RW, Strang V, Bennett-Clarke CA, Killackey HP, Chiaia NL (1997) Sensitive period for lesion-induced reorganization of intracortical projections within the vibrissae representation of rat's primary somatosensory cortex. J Comp Neurol 389:185-192.

Rice FL, Gomez C, Barstow C, Burnet A, Sands P (1985) A comparative analysis of the development of the primary somatosensory cortex: interspecies similarities during barrel and laminar development. J Comp Neurol 236:477-495.

Schlagger BL, O'Leary DDM (1994) Early development of the somatotopic map and barrel patterning in rat somatosensory cortex. J Comp Neurol 346:80-96.

Stojic AS, Lane RD, Killackey HP, Qadri BA, Rhoades RW (1998) Thalamocortical and intracortical projections to the forelimb-stump SI representation of rats that sustained neonatal forelimb removal. J Comp Neurol 401:187-204.

Wall PD, Egger MD (1971) Formation of new connections in adult rat brains after partial deafferentation. Nature 232:542-545.

Waters RS, McCandlish CA, Cooper NG (1990) Early development of SI cortical barrel subfield representation of forelimb in normal and deafferented neonatal rat as delineated by peroxidase conjugated lectin, peanut agglutinin (PNA). Exp Brain Res 81:234-240.

Waters RS, Li CX, McCandlish CA (1995) Relationship between the organization of the forepaw barrel subfield and the representation of the forepaw in layer IV of rat somatosensory cortex. Exp Brain Res 103:183-197.

Welker C (1976) Receptive fields of barrels in the somatosensory neocortex of the rat. J Comp Neurol 166:173-190.

Willis Jr WD, Coggeshall RE (1991) Sensory mechanisms of the spinal cord. New York: Plenum.

Wong-Riley MTT (1979) Changes in the visual system of monocularly sutured or enucleated cats demonstrable with cytochrome oxidase histochemistry. Brain Res 171:11-28.

Wong-Riley MTT, Welt C (1980) Histochemical changes in cytochrome oxidase of cortical barrels after vibrissal removal in neonatal and adult mice. Proc Natl Acad Sci USA 77:2333-2337.

Woolsey TA, Van der Loos H (1970) The structural organization of layer IV in the somatosensory region (SI) of mouse cerebral cortex. Brain Res 17:205-242.

Woolsey TA, Wann JR (1976) Areal changes in mouse cortical barrels following vibrissal damage at different postnatal ages. J Comp Neurol 170:53-66.

Woolsey TA, Welker C, Schwartz RH (1975) Comparative anatomical studies of the Sm1 face cortex with special reference to the occurrence of "barrels" in layer IV. J Comp Neurol 164:79-94.

Xu J, Wall JT (1999) Functional organization of tactile inputs from the hand in the cuneate nucleus and its relationship to organization in the somatosensory cortex. J Comp Neurol 411:369-389. 